\title{
Regulatory codewords
}

D eciphering the genome has been a metaphor and a research agenda for more than half a century. Indeed, an anniversary celebration for the genetic code is due, as it is just 50 years ago this month that Marshall Nirenberg presented the key piece of experimental evidence for the universal genetic code-a filter binding assay showing that the RNA trinucleotide codons pUpUpU, pApApA and pCpCpC could lock into the ribosome aminoacyl tRNAs carrying ${ }^{14} \mathrm{C}$-radiolabeled phenylalanine, lysine and proline, respectively (Science 145, 1399-1407, 1964). In this one paper, Nirenberg and Philip Leder not only offered the method to assign the codons of an mRNA to their respective amino acids in a peptide under translation but also identified the $5^{\prime}$ phosphate and $3^{\prime}$ hydroxyl groups on mRNA as the solution to the direction of the code. It is still quite inspiring to see them try to address then-unsolved problems of translational initiation, phasing and termination in terms of the chemistry of the ends of the mRNA molecule. Those testable speculations about the terminal codons were unsuccessful but did prefigure our current ideas of cap and tail modifications of mRNA, just as their concept of 'codeword modification' anticipated the roles of RNA editing.

Fast-forward through four decades of nucleic acid sequence determination (since the introduction of Sanger sequencing; J. Mol. Biol. 94, 441-446, 1975) and we have databases full of genome sequences from every kingdom of life, a very good idea of the conceptual proteins encoded by the open reading frames of their genes and quantitative models of the evolution of coding regions (see page 850 of this issue). Accumulation of hundreds of whole-genome sequences from cattle (page 858) and humans (page 818), alike analyzed by new bioinformatics pipelines for assembly and variant calling (page 912), readily yields prevalent coding mutations with immediate economic and medical implications.

And, of equal importance, this is also the fiftieth anniversary of the first publication (by François Jacob and colleagues using the lactose operon of Escherichia coli) to present simultaneous genetic analysis of a coding region, its cis-acting regulatory element and its regulatory protein (J. Mol. Biol. 8, 582-592, 1964). Today, after several decades of trapping enhancers and other genetic regulatory elements, we are on the verge of a new era of understanding in gene regulation at the genome level.

Admittedly, the 'regulatory code' is far from universal and the redundancy of its constituent sequences and DNA-binding proteins beggars that of the codons and their tRNAs. Our current model of an enhancer element regulating transcription in a eukaryote does not fit the metaphor of alphabet, cypher nor organelle and more closely resembles a flexible accretion of relatively independent transcription factor binding sites, often described as a 'billboard' (Development 130, 6569-6575, 2003 and Nat. Rev. Genet. 15, 453-468, 2014). This model is supported by systematic analysis of synthetic enhancer elements (Nat. Genet. 45, 1021-1028, 2013).

Although we are expecting synthetic biology and genomicscale enhancer trapping to establish much of the logic of noncoding elements (Nat. Genet. 46, 659, 2014), we would also welcome more speculative manuscripts modeling and offering testable predictions on the operation of gene-enhancer combinations and systems of genetic regulatory elements to stimulate research in this important field and to make the most of the genomic data we have harvested with the sequencers. 